\title{
Prevalence of Adverse Events to Radiopharmaceuticals from 2007 to 2011
}

\author{
Edward B. Silberstein \\ Departments of Radiology and Medicine, University of Cincinnati Medical Center, Cincinnati, Ohio
}

\begin{abstract}
We studied the changing patterns of radiopharmaceutical use and the incidence of adverse events (AEs) to PET radiopharmaceuticals, non-PET radiopharmaceuticals, and adjunctive nonradioactive pharmaceuticals in nuclear medicine from 2007 to 2011. Methods: Fifteen academic institutions submitted quarterly reports of radiopharmaceutical use and AEs covering 2007-2011. Results: $1,024,177$ radiopharmaceutical administrations were monitored: 207,281 diagnostic PET, 803,696 diagnostic non-PET, and 13,200 therapeutic. In addition, 112,830 adjunctive nonradioactive pharmaceutical administrations were monitored. The annual use of bone scintigraphy and radiotracer therapies was unchanged. PET radiopharmaceutical use increased from $17 \%$ to $26 \%$ of diagnostic procedures $(P<0.01)$. The incidence of radiopharmaceutical AEs was 2.1/10 administrations, with no hospitalizations or deaths. Conclusion: From 2007 to 2011, PET studies increased, and therapeutic radiopharmaceutical use and bone scintigraphy were unchanged. Over 2 decades, the incidence of AEs has remained stable at $2.1-2.3 / 10^{5}$ dosages.
\end{abstract}

Key Words: radiopharmaceuticals; adverse events; nuclear medicine safety

J Nucl Med 2014; 55:1308-1310

DOI: 10.2967/jnumed.114.138057

$\mathbf{I}$ is important to continuously monitor the use and adverse events (AEs) of radiopharmaceuticals to oversee, for our patients, our peers, and governmental regulators, the impressive safety record of our procedures (1-6), especially as new radiopharmaceuticals appear. We also inquired if there were changing patterns of use of radiopharmaceuticals for bone scintigraphy, PET, and radiolabeled antibody therapy for lymphoma.

\section{MATERIALS AND METHODS}

A group of nuclear pharmacists and physicians volunteered to join this unsponsored prospective study. These professionals are listed in the "Acknowledgments" section. The Institutional Review Board of the University of Cincinnati Medical Center ruled the study exempt from Institutional Review Board review according to title 45 of Code of Federal Regulations part 46.101 (b) (4). Nevertheless, some institutions

\footnotetext{
Received Jan. 25, 2014; revision accepted Apr. 22, 2014.

For correspondence contact: Edward B. Silberstein, University of Cincinnati Medical Center, 234 Goodman St., Cincinnati, OH 45219.

E-mail: edward.silberstein@uchealth.com

Published online May 29, 2014.

COPYRIGHT (c) 2014 by the Society of Nuclear Medicine and Molecular Imaging, Inc.
}

involved in the study did require Institutional Review Board review of the protocol, and approval was always granted.

To avoid the quandary of requiring strict proof of causality, we used the Food and Drug Administration definition of an AE: "Any untoward medical occurrence associated with the use of a drug in humans, whether or not considered drug related" (7-9). An AE algorithm relating to the probability of causation was carefully reviewed by all participants, who agreed to its use to establish the triple classification of AEs as probable, possible, or unlikely for radiopharmaceuticals and nonradioactive pharmaceuticals (5). All allergic, noxious, or unintended outcomes, signs, symptoms, and laboratory abnormalities were reported for radiopharmaceuticals. For nonradioactive pharmaceuticals, only AEs not previously reported in the medical literature-or those so serious that they led to hospitalization, were life-threatening, or were lethal-were to be reported, because tabulating well-documented AEs from nonradioactive pharmaceuticals would provide no new information. Types of AEs not within the scope of this study were excluded: altered biodistribution, vasovagal responses, deterministic and stochastic effects from therapeutic radiopharmaceuticals, overdoses, poor injection technique, or false-positive results (5). This AE algorithm has also been adopted by the Radiopharmacy Committee of the $\mathrm{Eu}-$ ropean Association of Nuclear Medicine (10).

The participants sent a quarterly report to the study coordinator over a 5-y period, 2007-2011, for all radiopharmaceuticals and nonradioactive pharmaceuticals used at their institutions, including those under a new drug application, investigational new drug application, or Radioactive Drug Research Committee supervision, and any radiopharmaceutical compounded on site. Any report of an AE was followed by a conversation with the coordinator, with joint agreement being achieved on the likelihood of causality for all AEs reported. Linear regression analysis was used to determine the significance of changes in the data points over time (Data Disk, version 6.3; Data Description, Inc.).

\section{RESULTS}

Fifteen institutions participated in the planning of this study, but only 13 , and finally 11 , could continue to contribute data for all $5 \mathrm{y}$, as a few institutions dropped out if the career or personal path of the reporter changed. From 2007 through 2011 the group reported on 1,010,977 diagnostic studies, of which $20.5 \%$ (207,281) represented PET studies and $79.5 \%(803,696)$ were studies with singlephoton-emitting radiopharmaceuticals, whether used for planar or SPECT scintigraphy. There were 13,200 therapeutic procedures, only $1.3 \%$ of the total of $1,024,177$ nuclear medicine procedures monitored for AEs. The percentage of therapeutic procedures per year ranged from $1.2 \%$ (2007) to $1.5 \%$ (2010) of the total, but there was no trend suggesting significantly increasing or decreasing numbers of therapies $(P>0.05)$. In addition, 112,830 adjunctive procedures with nonradioactive pharmaceuticals, comprising $11 \%$ of procedures with radiopharmaceuticals, were reported. 
TABLE 1

AE Results for 2007-2011

\begin{tabular}{|c|c|c|c|c|}
\hline Year & $\begin{array}{c}\text { Centers } \\
(n)\end{array}$ & $\begin{array}{c}\text { All AEs } \\
\text { (probable, } \\
\text { possible, } \\
\text { unlikely*) }\end{array}$ & Doses/y & $\begin{array}{c}\mathrm{AEs} / 10^{5} \\
\text { doses }\end{array}$ \\
\hline 2007 & 13 & 7 & 214,930 & 3.2 \\
\hline 2008 & 13 & 5 & 223,522 & 2.2 \\
\hline 2009 & 13 & 4 & 208,535 & 1.9 \\
\hline 2010 & 12 & 2 & 192,908 & 1.0 \\
\hline 2011 & 11 & 3 & 184,282 & 1.6 \\
\hline $5-y$ total & & 21 & $1,024,177$ & $2.1 \pm 0.6$ \\
\hline
\end{tabular}

*Five of these 21 AEs received a causality classification of unlikely.

\section{Trends in Radiopharmaceutical Use from 2007 to 2011}

There was a significant increase in PET studies as a percentage of the total over the $5 \mathrm{y}$ of the study, moving from $17 \%$ to $26 \%$ of all diagnostic studies $(P<0.01)$. The decrease in ${ }^{18} \mathrm{~F}-\mathrm{FDG}$ studies as a percentage of total PET scans from $85 \%$ to $80 \%$ was not statistically significant.

Labeled anti-CD20 antilymphoma antibodies have produced impressive levels of remission in refractory lymphoma and had been expected to have wide use, but this did not occur, as they represented $4.5 \%$ of therapies in 2007 and $4.0 \%$ in $2011(P>0.05)$, with a $5-y$ average of $3.3 \%$ of all therapies and no trend toward increasing or decreasing. The volume of single-photon bone scintigraphy, almost always with ${ }^{99 \mathrm{~m}} \mathrm{Tc}$-methylene diphosphonate, also remained constant, averaging $11.9 \%$ of all diagnostic nuclear medicine studies.

\section{AEs from Radiopharmaceuticals and Adjunctive Pharmaceuticals}

In Table 1, we have documented the annual number of AEs due to radiopharmaceuticals from 2007 to 2011 . The apparent decrease per $10^{5}$ administrations per year was not statistically significant. The decrease in absolute numbers of dosages reported per year
(Table 1) was caused by the loss of some investigators because of career or personal changes. Table 1 also provides the incidence of AEs (probable, possible, unlikely) per year from radiopharmaceuticals during the study, and the 21 AEs (including 5 deemed unlikely but that could not be excluded) are listed by symptom complex in Table 2. No AEs requiring hospitalization, deemed life-threatening, or lethal occurred.

\section{DISCUSSION}

The data collected in this study permit an examination of trends in nuclear medicine that might lead to a different pattern of radiopharmaceutical use, which had the potential to change for several reasons. Radiolabeled anti-CD20 antibodies have yielded unequivocal therapeutic advances $(11,12)$ but increased use did not occur, because of adequate results from the unlabeled antibody rituximab (13) and oncologist referral patterns.

PET has become an important diagnostic modality, and it was deemed possible that bone scintigraphic studies would diminish as a percentage of the total of diagnostic studies, since ${ }^{18} \mathrm{~F}$-FDG can detect tumor in marrow before the cortex is invaded (14). However, the number of bone scintigraphy procedures was stable over the study period. The volume of PET studies did rise. Other ${ }^{18} \mathrm{~F}$-labeled radiopharmaceuticals came into use (e.g., ${ }^{18} \mathrm{~F}$-sodium fluoride), potentially reducing the percentage of PET studies performed with ${ }^{18} \mathrm{~F}-\mathrm{FDG}$ PET, but the occurrence of this small change was not statistically significant. Although the estimated number of nuclear medicine procedures in the United States over the $5 \mathrm{y}$ of this study declined by about $9 \%$ (15), the number of procedures per institution in our study was essentially unchanged (16,533 in 2007 vs. 16,753 in 2011). Because we could not track changes in the use of over 40 radiopharmaceuticals, we do not have data that can more fully explain the use patterns observed.

Our primary goal was to document the incidence of AEs in the practice of nuclear medicine using prospective data collection by nuclear medicine scientists, clear definitions of AEs, $(16,17)$, and a known denominator $(16,17)$. With this approach, we believe we have overcome the problem of underreporting of AEs because of the transient nature of these events, confusion in the terminology of AEs, anxiety about potential liability, the time to complete a report form, and the lack of relevant reporting forms (16-19), although

TABLE 2

AEs Noted from Radiopharmaceuticals

\begin{tabular}{|c|c|}
\hline Event & Radiopharmaceutical \\
\hline Cutaneous (rash, flush) & 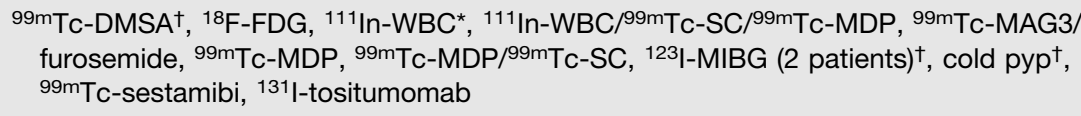 \\
\hline Nausea & ${ }^{123}$ I-MIBG $\left(2\right.$ patients) ${ }^{\dagger},{ }^{99 m}$ Tc-DMSA $^{\dagger}$ \\
\hline $\begin{array}{c}\text { Cardiovascular (anaphylactoid, } \\
\text { hypotension, cardiac arrest) }\end{array}$ & 99mTc-MDP (2 patients) ${ }^{\star},{ }^{99 m T c-S C},{ }^{18} \mathrm{~F}-\mathrm{FDG}^{*},{ }^{99 m} \mathrm{Tc}-\mathrm{MAG} 3 /$ furosemide \\
\hline $\begin{array}{l}\text { Neurologic (pain, hypesthesia, } \\
\text { paresthesia) }\end{array}$ & Cold pyp ${ }^{\dagger}, 99 \mathrm{~m} T \mathrm{c}-$ sestamibi*${ }^{*}, 99 \mathrm{~m} T \mathrm{c}$-tetrofosmin $\left(2\right.$ patients) ${ }^{\star}$ \\
\hline
\end{tabular}

\footnotetext{
* Judged as unlikely by study criteria, totaling 5 AEs; if 2 patients are noted as having had AEs, only one was deemed unlikely in this study.

†Three patients (1 each from ${ }^{99 m}$ Tc-DMSA, ${ }^{123}$ I-MIBG, cold pyp) had 2 symptoms or signs from radiopharmaceuticals, but these were counted as $1 \mathrm{AE}$ from 1 radiopharmaceutical that caused 2 symptoms.

DMSA = dimercaptosuccinic acid; WBC = white blood cells; $\mathrm{SC}=$ sulfur colloid; MDP = methylene diphosphonate; MAG3 = mercaptoacetyltriglycine; MIBG = metaiodobenzylguanidine; pyp = pyrophosphate.
} 
MedWatch, the Safety Information and Adverse Event Reporting Program of the Food and Drug Administration, is available online at www.fda.gov/Safety/MedWatch/.

Because diagnostic radiopharmaceuticals are, by definition, not given for therapeutic purposes, one would expect few physiologic effects or AEs from them if the specific activity of these radiotracers is sufficiently high. Therefore, it is hardly surprising that such AEs are quite uncommon. In 1996 a survey study (covering 1989-1994) showed an AE incidence of $2.3 / 10^{5}$ dosages (5), and in this current study we have reported a virtually identical finding, 2.1 AEs $/ 10^{5}$ administrations. Deterministic effects of therapeutic radiopharmaceuticals are not infrequent because of the activity of radiation deposited at sites of their normal physiologic distribution (e.g., ${ }^{131}$ I gastritis, sialadenitis, oral mucositis), but no therapeutic radiopharmaceutical (13,200 administrations) or interventional nonradioactive drug (112,830 administrations) in this study caused hospitalization, a life-threatening $\mathrm{AE}$, or death.

Outside our study, 2 deaths and 15 life-threatening AEs followed administration of the anti-CD15 antibody ${ }^{99 \mathrm{~m}} \mathrm{Tc}$-fanolesomab (NeutroSpec; Palatin Technologies), introduced in 2004 and withdrawn from the market in December 2005. No other deaths from radiopharmaceuticals have been reported since 1975 except for two from an albumin colloid and one from diethylenetriaminepentaacetic acid briefly mentioned and undated in a 1993 review (20).

In the current study, we report the first (to our knowledge) AEs from ${ }^{18} \mathrm{~F}-\mathrm{FDG}$, flushing of the face and trunk occurring within minutes of administration and lasting less than $2 \mathrm{~h}$ after injection. Other AEs not previously reported occurred with ${ }^{99 \mathrm{~m}} \mathrm{Tc}$-labeled dimercaptosuccinic acid, sestamibi, and tetrofosmin (Table 2).

There are potential weaknesses of this study. The institutions in this study may not represent the practice of nuclear medicine elsewhere, although most radiopharmaceuticals should be the same. Also, minor AEs could have been missed or ignored by the nuclear medicine technologist. We chose to include in our report all AEs, including those believed to be unlikely, since, importantly, the "unlikely" label also fits any AE on its first occurrence. Nevertheless, the results from this and our previous studies $(5,6)$ are virtually identical and support the credibility of these results, using the definitions and methodology described above.

\section{DISCLOSURE}

The costs of publication of this article were defrayed in part by the payment of page charges. Therefore, and solely to indicate this fact, this article is hereby marked "advertisement" in accordance with 18 USC section 1734 . No potential conflict of interest relevant to this article was reported.

\section{ACKNOWLEDGMENTS}

The collaboration and wisdom of the following contributors (and their institutions) is deeply appreciated: Michelle Beauvais (William Beaumont Medical Center), Neil Petry (Duke Medical Center), Richard Reba (Georgetown Medical Center), James Ponto (Iowa University Medical Center), Steven Mattmuller (Kettering
Medical Center, Dayton), Steven Karsh (Loyola Medical Center), Sandra Stoops (University of Maryland Medical Center), Joseph Hung (Mayo Clinic), Allegra Bruce (Massachusetts General Hospital), Ronald Weiner (National Institutes of Health), Richard Kowalsky (University of North Carolina Medical Center), Scott Knishka (University of Wisconsin Medical Center), Robert Massey (Walter Reed Medical Center), and Sally Schwarz (Washington University Medical Center). The statistical consultation of Chandrasiri Samaratunga is gratefully appreciated.

\section{REFERENCES}

1. Ford L, Shroff A, Benson W, Atkins HL, Rhodes BA. Adverse reactions to radiopharmaceuticals. J Nucl Med. 1978;19:116-117.

2. Rhodes BA, Cordova MA. Adverse reactions to radiopharmaceuticals: incidence in 1978 and associated symptoms-report of the Adverse Reactions Subcommittee of the Society of Nuclear Medicine. J Nucl Med. 1980;21:1107-1110.

3. Cordova MA, Rhodes BA, Atkins HL, et al. Adverse reactions to radiopharmaceuticals. J Nucl Med. 1982;23:550-551.

4. Atkins HL. Reported adverse reactions to radiopharmaceuticals remain low in 1984. J Nucl Med. 1986;27:327.

5. Silberstein EB, Ryan J. Prevalence of adverse reactions in nuclear medicine. Pharmacopeia Committee of the Society of Nuclear Medicine. J Nucl Med. 1996; 37:185-192, 1064-1067.

6. Silberstein EB. Prevalence of adverse reactions to positron emitting radiopharmaceuticals in nuclear medicine. Pharmacopeia Committee of the Society of Nuclear Medicine. J Nucl Med. 1998;39:2190-2192.

7. U.S. Department of Health and Human Services website. IND safety reporting: definitions. 21 CFR §312.32(a). http://www.accessdata.fda.gov/scripts/cdrh/cfdocs/ cfcfr/cfrsearch.cfm?fr=312.32. Revised April 1, 2013. Updated June 6, 2013. Accessed May 7, 2014.

8. Venulet J, Ciucci AG, Berneker G-C. Updating of a method for causality assessment of adverse drug reactions. Int J Clin Pharmacol Ther Toxicol. 1986;24:559-568.

9. Hutchinson TA, Leventhal JM, Kramer MS, Karch FE, Lipman AG, Feinstein AR. An algorithm for the operational assessment of adverse drug reactions: II. Demonstration of reproducibility and validity. JAMA. 1979;242:633-638.

10. Hesslewood SR, Keeling DH. Frequency of adverse reactions to radiopharmaceuticals in Europe. Eur J Nucl Med. 1997;24:1179-1182.

11. Seiler T, Hiddemann W, Dreyling M. Optimal application of antibodies in the treatment of follicular lymphoma: current standards and future strategies. Immunotherapy. 2009;1:1015-1024.

12. Bodet-Milin C, Ferrer L, Pallardy A, et al. Radioimmunotherapy of B-cell nonHodgkin's lymphoma. Front Oncol. 2013;3:177-186.

13. Okamoto A, Yanada M, Inaguma Y, et al. Differences in outcome for consecutive patients with diffuse large B-cell lymphoma before and after the advent of rituximab: a single-center experience. Hematology. 2013;18:74-80.

14. Lee JW, Lee SM, Lee HS, Kim YH, Bae WK. Comparison of diagnostic ability between ${ }^{99 \mathrm{~m} T c-M D P}$ bone scan and ${ }^{18} \mathrm{~F}-\mathrm{FDG}$ PET/CT for bone metastasis in patients with small cell lung cancer. Ann Nucl Med. 2012;26:627-633.

15. IMV Medical Information Division. Nuclear Medicine Market Outlook Report. Imaging Technology News. December 26, 2013.

16. Pintor-Mármol A, Baena IB, Fajardo PC, et al. Terms used in patient safety related to medication: literature review. Pharmacoepidemiol Drug Saf. 2012;21: 799-809.

17. Safety of medicines: a guide to detecting and reporting adverse drug reactions — why health professionals need to take action. World Health Organization website. http:// apps.who.int/medicinedocs/en/d/Jh2992e/. Published 2002. Accessed May 7, 2014.

18. Hesse B, Vinberg N, Berthelsen AK, Ballinger JR. Adverse events in nuclear medicine: cause for concern? Eur J Nucl Med Mol Imaging. 2012;39:782-785.

19. Salvatori M, Treglia G, Mores N. Further considerations on adverse reactions to radiopharmaceuticals. Eur J Nucl Med Mol Imaging. 2012;39:1360-1362.

20. Sampson CB. Adverse reactions and drug interactions with radiopharmaceuticals. Drug Saf. 1993;8:280-294. 